\title{
Guarded Computational Type Theory
}

\author{
Jonathan Sterling \\ Computer Science Department \\ Carnegie Mellon University \\ jmsterli@cs.cmu.edu
}

\author{
Robert Harper \\ Computer Science Department \\ Carnegie Mellon University \\ rwh@cs.cmu.edu
}

\begin{abstract}
Nakano's later modality can be used to specify and define recursive functions which are causal or synchronous; in concert with a notion of clock variable, it is possible to also capture the broader class of productive (co)programs. Until now, it has been difficult to combine these constructs with dependent types in a way that preserves the operational meaning of type theory and admits a hierarchy of universes $\mathrm{U}_{i}$.

We present an operational account of guarded dependent type theory with clocks called $\mathbf{C T T}_{\odot}$, featuring a novel clock intersection connective $\{k \div \mathrm{clk}\} \rightarrow A$ that enjoys the clock irrelevance principle, as well as a predicative hierarchy of universes $U_{i}$ which does not require any indexing in clock contexts. $\mathbf{C T T}_{\odot}$ is simultaneously a programming language with a rich specification logic, as well as a computational metalanguage that can be used to develop semantics of other languages and logics.
\end{abstract}

CCS Concepts - Theory of computation $\rightarrow$ Type theory; Modal and temporal logics;

Keywords guarded recursion, clocks, type theory, operational semantics, dependent types

ACM Reference Format:

Jonathan Sterling and Robert Harper. 2018. Guarded Computational Type Theory. In LICS '18: LICS '18: 33rd Annual ACM/IEEE Symposium on Logic in Computer Science, July 9-12, 2018, Oxford, United Kingdom. ACM, New York, NY, USA, 10 pages. https://doi.org/10.1145/3209108.3209153

\section{Introduction}

In a functional programming language, every definable function is continuous in the following sense: each finite quantity of output is induced by some finite quantity of input. To make this more precise, if we consider the case of stream transformers $F: \mathbb{S} \rightarrow \mathbb{S}$, we can see that finite prefixes of the output depend only on finite prefixes of the input:

$$
\forall \alpha: \mathbb{S} . \forall i: \mathbb{N} . \exists n: \mathbb{N} . \forall \beta: \mathbb{S} . \alpha \equiv_{n} \beta \Rightarrow F(\alpha)_{i} \equiv F(\beta)_{i}
$$

From a programming perspective, this can be rephrased in terms of reads and writes: for each write, the program is permitted to perform a finite but unbounded number of reads.

Permission to make digital or hard copies of all or part of this work for personal or classroom use is granted without fee provided that copies are not made or distributed for profit or commercial advantage and that copies bear this notice and the full citation on the first page. Copyrights for components of this work owned by others than the author(s) must be honored. Abstracting with credit is permitted. To copy otherwise, or republish, to post on servers or to redistribute to lists, requires prior specific permission and/or a fee. Request permissions from permissions@acm.org.

LICS '18, July 9-12, 2018, Oxford, United Kingdom

(c) 2018 Copyright held by the owner/author(s). Publication rights licensed to Association for Computing Machinery.

ACM ISBN 978-1-4503-5583-4/18/07 ..\$15.00

https://doi.org/10.1145/3209108.3209153
Causality Another possible class of functionals are the ones that can be implemented by a program which performs at most one read for every write. These are called the causal functionals, and in the case of stream transformers, they are characterized by the following causality principle:

$$
\forall \alpha: \mathbb{S} . \forall i: \mathbb{N} . \forall \beta: \mathbb{S} . \alpha \equiv_{i} \beta \Rightarrow F(\alpha)_{i} \equiv F(\beta)_{i}
$$

In other words, causal programs are the ones whose reads and writes proceed in lock-step. While we can surely carve out this class of functionals using predicates like (2) above, it is actually possible to define a new notion of stream $\mathbb{S}$ such that all functionals $F$ : $\mathbb{S}_{\downarrow} \rightarrow \mathbb{S}_{\downarrow}$ are automatically causal in the sense of (2). This kind of stream is called a "guarded stream", and we will use the term "sequence" to refer to ordinary streams.

Whereas ordinary streams or sequences are usually formed as the greatest solution to the isomorphism $\mathbb{S} \cong \mathbb{N} \times \mathbb{S}$, the guarded streams are formed using a special "later modality" $\longrightarrow$ due to Nakano, ${ }^{1}$ solving the isomorphism $\mathbb{S} \cong \mathbb{N} \times \mathbb{S} \downarrow$. Modalities of this kind usually enjoy at least the following principles:

$$
\begin{array}{cl}
A \rightarrow \neg A \quad \triangleright(A \times B) \cong(\triangleright A \times \triangleright B) \\
\triangleright(A \rightarrow B) \rightarrow(\triangleright A \rightarrow \triangleright B) \quad(\triangleright A \rightarrow A) \rightarrow A
\end{array}
$$

The ratio of reads and writes specified in the type of a stream transformer can be modulated by adjusting the number of later modalities in the input and the output to the function.

Nakano's modality in semantics What is remarkable about Nakano's later modality is that fixed points for functions $F: \triangle A A$ always exist, without placing any restriction on $F$ (such as monotonicity or positivity). Applied within a type-theoretic metalanguage, then, the later modality induces solutions to recursive domain equations which are not set-theoretically interpretable, such as the following classic definition of semantic types for a programming language with mutable store $[5,9]$ :

$$
\mathcal{T}_{y p e} \cong(\mathcal{L} \text { oc } \stackrel{\text { fin }}{\longrightarrow} \triangleright \mathcal{T} \text { ype }) \rightarrow \mathcal{P}(\mathcal{V a l})
$$

The later modality captures and internalizes the basic features of less abstract techniques like step-indexing, enabling more streamlined definitions and proofs that elide the bureaucratic performance of explicit indexing and monotonicity obligations. Today, modalities of this kind are of the essence for modern program logics like Iris [24].

Programming applications The fact that functions $F: A \rightarrow A$ always have fixed points has beneficial consequences for the practice of (total) functional programming on infinite data. In particular, clumsy syntactic guardedness conditions which ensure productivity (such as those used in Coq [35], Agda [29] and Idris [15]) can be

\footnotetext{
${ }^{1}$ The notation • was originally used in Nakano [28].
} 


$$
\begin{aligned}
& \frac{\Delta, \kappa ; \Gamma \vdash e: A}{\Delta ; \Gamma \vdash \Lambda \kappa \cdot e: \forall \kappa \cdot A} \quad \frac{\kappa^{\prime} \in \Delta \quad \begin{array}{c}
\Delta ; \Gamma \vdash e: \forall \kappa \\
\kappa^{\prime} \notin \operatorname{FreeClocks}(A)
\end{array}}{\Delta ; \Gamma \vdash e\left[\kappa^{\prime}\right]: A\left[\kappa \hookrightarrow \kappa^{\prime}\right]}
\end{aligned}
$$

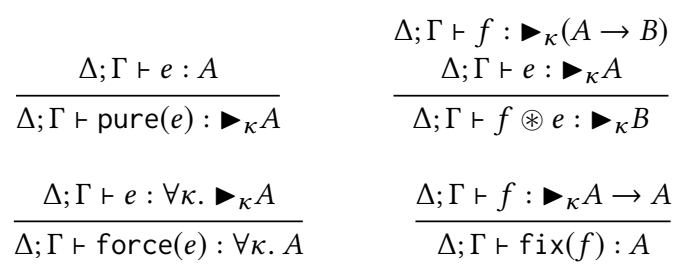

$$
\begin{aligned}
& (\forall \kappa . A) \equiv A \quad(\kappa \notin \text { FreeClocks }(A)) \\
& \forall \kappa . A \times B \equiv(\forall \kappa . A) \times(\forall \kappa . B)
\end{aligned}
$$

Figure 1. Selection of rules from Atkey and McBride [6].

replaced with type structure, enabling more compositional styles of programming. ${ }^{2}$

However, the later modality is too restrictive to be used on its own, because it rules out the functions which are not causal; but acausal functions on infinite data are perfectly sensible, and are very common in the real world. Consider, for instance, the function which drops every second element from a stream! To define this function, one would need a way to delete the modality; but without suitable restrictions, such an elimination principle would trivialize the modality and render it useless.

To resolve this problem, Atkey and McBride have introduced a notion of abstract clock $\kappa$ to represent "time streams" together with universal quantification $\forall \kappa$ over clocks, replacing Nakano's modality with a clock-indexed family of modalities $\nabla_{\kappa}[6]$.

Defining the type of $\kappa$-guarded streams as the solution to the equation $\mathbb{S}_{\kappa} \equiv \mathbb{N} \times{ }_{\kappa} \mathbb{S}_{\kappa}$, it is possible to define the acausal function that drops every other element of a stream, with type $\left(\forall \kappa . \mathbb{S}_{\kappa}\right) \rightarrow\left(\forall \kappa . \mathbb{S}_{\kappa}\right)$. The reason that this is possible is that their calculus exhibits the isomorphism $\left(\forall \kappa .{ }_{\kappa} A\right) \cong(\forall \kappa . A)$, as well as a clock irrelevance principle: $(\forall \kappa . A) \equiv A$ assuming that $\kappa$ is not free in $A$; we summarize the constructs of this calculus in Figure 1.

\subsection{Dependent type theory and guarded recursion}

It has been surprisingly difficult to cleanly extend the account of guarded recursion with clocks to a full-spectrum dependently typed programming language which enjoys any combination of the following properties:

1. Computational canonicity: any closed element of type bool computes to either $t \mathrm{t}$ or $\mathrm{ff}$.

2. Simple universes: a single predicative and cumulative hierarchy of universes $U_{i}$ closed under base types, dependent function types, dependent pair types, lower universes, later modalities and clock quantifiers.

3. Clock irrelevance: if $k$ is not mentioned in $A$ and $A$ is a type, then $\forall k$. $A$ is equal to $A^{3}$

\footnotetext{
${ }^{2}$ A very closely related idea, sized types, has been deployed in the Agda proof assistant for exactly this purpose [36].

${ }^{3}$ Depending on the specific type theory, it may be desirable to realize this principle either as an isomorphism or as a definitional equality.
}

However, a dependent type theory with support for guarded recursion and clocks is desirable for multiple reasons; here, we have focused on causality as a useful construct for developing types qua behavioral specifications on program behavior, but there is also the potential to use such a dependent type theory as a computational metalanguage for developing and proving the semantics of other languages and logics, vaporizing the highly-bureaucratic stepindexed Kripke Logical Relations which usually must be employed.

The latter perspective is elaborated in the context of guarded dependent type theory without clocks in Paviotti et al. [30] as well as Bizjak et al. [11], and we anticipate that the addition of clocks will enable further developments along these lines.

\subsection{Guarded Computational Type Theory}

We contribute a new extensional and behavioral dependent type theory CTT $\odot$ (pronounced "Guarded Computational Type Theory") for guarded recursion and clocks in the Nuprl tradition [2], enjoying the following characteristics:

1. Operational semantics and an immediate canonicity result at base types.

2. A clock-indexed later modality ${ }_{k} A$ which requires no special syntax for introduction or destruction.

3. A decomposition of the clock quantifier from Bizjak and Møgelberg [14] into a parametric part $\{k \div \mathrm{clk}\} \rightarrow A$ and a non-parametric part $(k: \mathrm{clk}) \rightarrow A$. The former is an intersection, and enjoys the crucial clock irrelevance principle; the latter is the cartesian product of a clock-indexed family of sets (right adjoint to weakening).

4. A guarded fixed point combinator which can be assigned the type $\left(\nabla_{k} A \rightarrow A\right) \rightarrow A$.

5. A predicative hierarchy of universes $U_{i}$ closed under all the connectives, free of indexing by clock contexts.

Our operational account and canonicity result (Theorem 7) means that $\mathbf{C T T}_{\odot}$ can be regarded simultaneously as a programming language with a rich specification logic, and as a computational metalanguage for developing operational and denotational semantics of other languages and logics.

\section{Coq formalization and synthetic approach}

Using the Coq proof assistant, we have formalized the fragment of our type theory that contains universes, dependent function and pair types, booleans, the later modality, and the two clock quantifiers (intersection and product); the full Coq development is available in Sterling and Harper [34]. Throughout this paper, theorems and rules will be related to their Coq analogues using a reference like Module. theorem_name.

We have used Coq's type theory as a proxy for the internal language of the presheaf topos that we develop herein, axiomatizing in Coq whatever objects and principles come not from the standard type theoretic constructions, but are instead imported into the system via forcing. The entire construction of $\mathbf{C T} \mathbf{T}_{\odot}$, then, is carried out within the internal language of the topos, an anti-bureaucratic measure which has made an otherwise daunting formalization effort feasible.

The idea of developing operational models of programming languages within the internal language of a topos is not new; see for instance Staton [32], Bizjak et al. [11] and Paviotti et al. [30]. However, we believe that ours is the first instance of this technique being 
applied toward the development of semantics for a full-spectrum dependent type theory.

Full details and proofs of our construction can be found in our report [33].

\section{Programming in $\mathrm{CTT}_{\odot}$}

Following the computational meaning-theoretic tradition initiated by Martin-Löf [26], and developed further in the Nuprl project [2], we build Guarded Computational Type theory $\left(\mathbf{C T T}_{\odot}\right)$ on the basis of an untyped programming language, whose syntax is summarized in Figure 2.

In this paper, we distinguish between the syntax of formal terms and the language of programs; formal terms are used by clients of a formalism for type theory, whereas programs are the things which are actually endowed with operational meaning. For many languages, the difference between formal terms and programs is not so great, but for us the difference is essential; to avoid confusion, we distinguish between these levels using colors.

Formal Terms The grammar includes operators for both terms and types, which are not distinguished syntactically in any way. Typehood, equality and type membership are semantic properties which will be imposed after we propound the meaning explanation in Section 3.6. We include syntax for dependent function types $(x: A) \rightarrow B$, dependent pair types $(x: A) \times B$, wellordering types $\mathrm{W}(x: A) B$, extensional equality types $\mathrm{Eq}_{A}(M ; N)$, clockindexed later modalities $\nabla_{k} A$, clock product types $(k: \mathrm{clk}) \rightarrow A$, clock intersection types $\{k \div \mathrm{clk}\} \rightarrow A$, booleans, natural numbers, and a countable hierarchy of type universes $U_{i}$. We define the following derived forms for non-dependent function and pair types:

$$
A \rightarrow B \triangleq(x: A) \rightarrow B \quad A \times B \triangleq(x: A) \times B
$$

Forming fixed points and primitive recursors General fixed points can be programmed in $\mathbf{C T T}_{\odot}$ exactly as in the untyped $\lambda$-calculus, but in order to simplify our metatheorems we have provided a primitive fixed point operator fix $x$ in $M$. This can, for instance, be used to realize the induction principle for the natural numbers.

When a function has type ${ }_{\kappa} A \rightarrow A$, its guarded fixed point always exists and has type $A$. Because $\mathbf{C T T}_{\odot}$ is dependently typed, it is very easy for us to write a program that computes the type of guarded streams of bits relative to a clock $k$, using the fixed point operator in concert with the later modality; and using the clock intersection type, we can transform this into the type of infinite sequences of bits:

$$
\begin{aligned}
& \text { stream } \in(k: \mathrm{clk}) \rightarrow \mathrm{U}_{i} \\
& \text { stream } \triangleq \lambda k . \mathrm{fix} A \text { in bool } \times{ }_{k} A \\
& \text { sequence } \in \mathrm{U}_{i} \\
& \text { sequence } \triangleq\{k \div \mathrm{clk}\} \rightarrow \text { stream } k
\end{aligned}
$$

We will see in Section 3.8 that these expressions are indeed types in $\mathrm{CTT}_{\odot}$.

\section{Mathematical Meaning Explanation}

In the type-theoretic tradition of Martin-Löf, formal language is endowed with computational meaning through what is called a "meaning explanation"; this style of definition, which was first deployed by Martin-Löf in his seminal paper Constructive Mathematics and Computer Programming [26], is closely related to PER semantics and the method of computability. This computational perspective was developed to its fullest extent in Nuprl's CTT [2, 18], which adds a theory of computational congruence to the picture, together with many new connectives including intersections, unions, subset comprehensions, quotients and image types.

A meaning explanation provides a semantics for types as specifications of the execution behavior of untyped programs. As such, the judgments of type theory express the compliance of a program with a specification, which can be of arbitrary quantifier complexity, and will not generally be decidable. Any implementation of type theory involves, in one form or another, a formal system for deriving correct judgments that is, by definition, recursively enumerable and often decidable.

To achieve various properties that are desirable of a formal system (sometimes including decidability), programs are often decorated with type information that is not needed during execution. The meaning explanation is, then, lifted to the formalism along an erasure map $\|-\|$ that removes these decorations.

A similar, but more elaborate transformation of syntax (from formal terms to programs) is used here to facilitate the meaning explanation for guarded type theory in terms of the settings of a collection of clocks. During the verification of a program specification, the value of a clock may change (for instance, underneath the later modality); the most direct way to express this is to explicitly formulate the meaning explanation using a Kripke or presheaf-style semantics: a "possible world" consists of a collection of clocks and their settings, and we require specifications to account for the expansion of the world with new clocks and the alterations of their settings.

Doing so tends to clutter the meaning explanation by distributing the conditioning on clocks throughout the semantics, and disrupts a basic principle of type theory in the Martin-Löf tradition, which is that types should do little more than internalize the structures which are already present in the judgmental base.

An alternative, which we adopt here, is to formulate the semantics in a presheaf topos $\mathcal{S}_{\odot}$ which accounts all at once for clocks and the passage of time, so that the specifications given by types are implicitly conditioned on them. This conditioning, which is implicit when viewed from inside the topos, can be externalized and made explicit using the Kripke-Joyal forcing semantics of $\mathcal{S}_{\odot}$ [25].

To ensure that programs evolve appropriately along the transitions between clock worlds simultaneously with their specifications, we introduce a kind of "higher-order abstract syntax" which links clocks in programs directly to their meaning in the presheaf topos, as elements of the presheaf of clocks $\mathbb{K}: \mathcal{S}_{\odot}$. The passage to this new kind of syntax at the interface between the formalism and the semantics is managed by an elaboration function $\|-\|$.

\subsection{The semantic universe $\mathcal{S}_{\odot}$}

We will develop our semantic universe as a presheaf topos called $\mathcal{S}_{\odot}$ over a category of clock contexts and clock context morphisms. We will require the following things to exist in $\mathcal{S}_{\odot}$ :

1. An object $\mathbb{K}: \mathcal{S}_{\odot}$ of clock names.

2. A family of logical modalities $\triangleright_{\kappa} \phi$ for clock names $\kappa: \mathbb{K}$ and predicates $\phi$ in $\mathcal{S}_{\odot}$. 


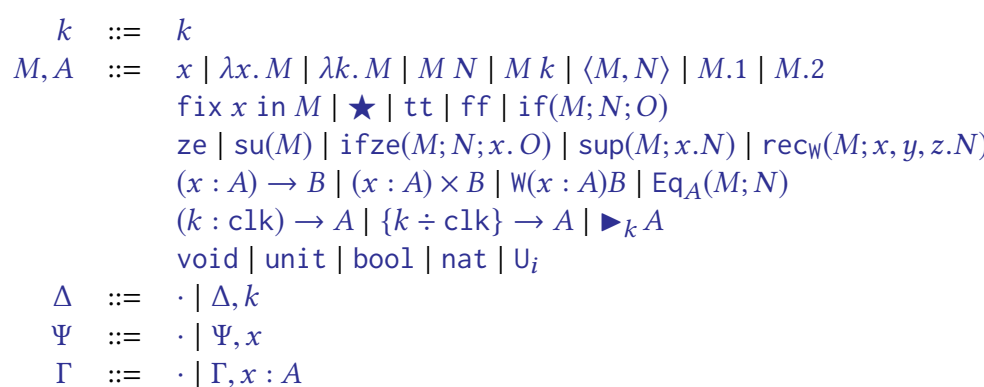

(clocks)

(terms)

(clock contexts)

(variable contexts)

(typing contexts)

Figure 2. The syntax of formal terms in Guarded Computational Type Theory $\left(\mathbf{C T T}_{\odot}\right)$. Formal terms $M$ are identified up to renamings of their bound variables; by convention, bound variables are always assumed fresh.

When we define $\mathcal{S}_{\odot}$, we will arrange for the following principles to hold in its internal logic:

$$
\begin{aligned}
& \exists \kappa: \mathbb{K} . \top \\
& \forall \phi: \Omega^{\mathbb{K}} \cdot\left(\forall \kappa: \mathbb{K} . \triangleright_{\kappa} \phi(\kappa)\right) \Rightarrow \forall \kappa: \mathbb{K} . \phi(\kappa) \\
& \forall \kappa: \mathbb{K} . \forall \phi: \Omega . \phi \Rightarrow \triangleright_{\kappa} \phi \\
& \forall \kappa: \mathbb{K} . \forall \phi, \psi: \Omega . \triangleright_{\kappa}(\phi \wedge \psi) \equiv\left(\triangleright_{\kappa} \phi \wedge \triangleright_{\kappa} \psi\right) \\
& \forall \kappa: \mathbb{K} . \forall \phi, \psi: \Omega . \triangleright_{\kappa}(\phi \Rightarrow \psi) \equiv\left(\triangleright_{\kappa} \phi \Rightarrow \triangleright_{\kappa} \psi\right) \\
& \forall \kappa: \mathbb{K} . \forall \phi: \Omega .\left(\triangleright_{\kappa} \phi \Rightarrow \phi\right) \Rightarrow \phi
\end{aligned}
$$

We require one additional axiom to hold for any object $Y: \mathcal{S}_{\odot}$ which is total and inhabited in a sense analogous to the notion from Birkedal et al. [9]:

$$
\forall \kappa: \mathbb{K} . \forall \phi: \Omega^{Y} . \triangleright_{\kappa}(\exists y: Y . \phi(y)) \Rightarrow \exists y: Y .{ }_{\kappa} \phi(y)
$$

To construct $\mathcal{S}_{\odot}$ as a topos of presheaves, first define $\mathbb{F}_{+}$: Cat as the free category with strictly associative binary products generated by a single object; explicitly, objects of $\mathbb{F}_{+}$are $U \equiv \bullet^{n}$ for $n>0$. A map $f: \bullet^{n} \rightarrow \bullet^{m}$ is a vector of projections, but can dually be regarded as a function between finite sets $\mathbb{N}_{<m} \rightarrow \mathbb{N}_{<n}$.

Observe that the opposite category $\mathbb{F}_{+}^{\text {op }}$ is a skeleton of the category of non-empty finite sets and all functions between them. $\mathbb{F}_{+}$is also a full subcategory of $\mathbb{F}$ : Cat, the free strict cartesian category generated by a single object (whose opposite is likewise a skeleton of the category of finite sets and all maps between them).

Define the presheaf of clock names $\mathcal{N}: \widehat{\mathbb{F}_{+}}$as the representable functor $\mathrm{y}\left(\bullet^{1}\right)$. Next, define a functor $\odot[-]: \mathbb{F}_{+} \rightarrow$ Pos (with Pos the category of partially ordered sets) which will interpret assignments of times to clock names:

$$
\begin{aligned}
& \text { () }[-]: \mathbb{F}_{+} \rightarrow \text { Pos } \\
& \odot\left[U: \mathbb{F}_{+}\right] \triangleq \omega^{\mathcal{N}(U)} \\
& \odot[f: V \rightarrow U]\left(\partial_{V}: \omega^{\mathcal{N}(V)}\right) \triangleq(\kappa: \mathcal{N}(U)) \mapsto \partial_{V}\left(f^{*} \kappa\right)
\end{aligned}
$$

Thinking of elements of $\mathbb{F}_{+}$as signifying finite and non-empty cardinalities of clock names, the action of $\odot[-]$ on objects takes such a cardinality $U: \mathbb{F}_{+}$to the $U$-fold product of the poset $\omega$, ordered pointwise: in other words, it assigns the amount of "time left" to each clock.

Finally, using the covariant Grothendieck construction [20] we can build the total category $\odot$ : Cat $\triangleq \int^{\mathbb{F}_{+}} \odot[-]$ in the following way. Objects are pairs $\left(U: \mathbb{F}_{+}, \partial_{U}: \odot[U]\right)$, i.e. collections of clock names together with an assignment; morphisms $f:\left(V, \partial_{V}\right) \rightarrow\left(U, \partial_{U}\right)$ are $\mathbb{F}_{+}$-morphisms $f: V \rightarrow U$ such that $\odot[f]\left(\partial_{V}\right) \leq \partial_{U}$ in $\odot[U]$.
At this time it will be helpful to impose some notation: we will write $\ell: \odot \rightarrow \mathbb{F}_{+}$for the induced projection functor, and we will use boldface letters $\mathrm{U}, \mathrm{V}$ to range over objects $\left(U, \partial_{U}\right),\left(V, \partial_{V}\right):$ (๑) .

The semantic universe $\mathcal{S}_{\odot} \quad$ Finally, we define our semantic universe as the presheaf topos $\mathcal{S}_{\odot} \triangleq \widehat{\Theta}$. This "topos of clocks" defined above inherits a rich internal logic which corresponds to a combination of cartesian/structural nominal $\operatorname{logic}^{4}$ and guarded recursion.

The topos $\mathcal{S}_{\odot}$ is related to the models considered by Bizjak and Møgelberg [13], except that rather than constructing a family of presheaf toposes fibered over clock contexts, we combine clock contexts with time assignments into a single base category, and take the topos of presheaves over that; our topos is nearly identical to the presheaf category considered independently in Bizjak and Møgelberg [14].

One minor difference between our model and those of Bizjak and Møgelberg is that in order to close the internal logic of $\mathcal{S}_{\odot}$ under the clock irrelevance axiom described above, we decided to rule out empty clock contexts; this condition is equivalent to taking a sheaf subtopos of the presheaves over all clock contexts.

The object of clock names We need to exhibit an object in the presheaf topos $\mathcal{S}_{\odot}$ whose elements are the "available" clock names (without regard to their time assignments). First observe that the representable object $\mathcal{N}$ plays exactly this role in the category $\widehat{\mathbb{F}_{+}}$: at clock context $\bullet^{n}$ it consists in the set of morphisms $\bullet^{n} \rightarrow \bullet^{1}$, which has cardinality $n$. However, this object resides in the wrong topos, since we need to define an object $\mathbb{K}: \mathcal{S}_{\odot}$. To achieve this, we use the reindexing functor $\ell^{*}: \widehat{\mathbb{F}_{+}} \rightarrow \mathcal{S}_{\odot}$ induced by precomposing the projection $\ell: \mathcal{S}_{\odot} \rightarrow \mathbb{F}_{+}$, defining $\mathbb{K} \triangleq \ell^{*} \mathcal{N}$.

Notations and morphisms We write $\mathrm{U}[\kappa \mapsto n]$ to mean $\left(U, \partial_{U}[\kappa \mapsto\right.$ $n]$ ), where $\partial_{U}[\kappa \mapsto n]$ means the adjustment to $\partial_{U}$ which replaces $\partial_{U}(\kappa)$ with $n$. Finally, for the map that increments the time assigned to a clock, we write $[\kappa+=1]: \mathbf{U} \rightarrow \mathbf{U}\left[\kappa \mapsto \partial_{U}(\kappa)+1\right]$.

Defining the $\triangleright_{\kappa}$ modalities We define the $\triangleright_{\kappa}$ modalities by their forcing clause in the Kripke-Joyal semantics of $\mathcal{S}_{\odot}:{ }^{5}$

$$
\mathrm{U} \Vdash \triangleright_{\kappa} \phi(\alpha) \triangleq \begin{cases}\top & \text { if } \partial_{U}(\kappa) \equiv 0 \\ \mathrm{U}[\kappa \mapsto n] \Vdash \phi\left([\kappa+=1]^{*} \alpha\right) & \text { if } \partial_{U}(\kappa) \equiv n+1\end{cases}
$$

\footnotetext{
${ }^{4}$ That is, the logic of nominal substitution sets [22, 32].

${ }^{5}$ Usually the forcing clauses should be taken as theorems rather than as definitions. However, in a Grothendieck topos, it is possible to define a subobject by its forcing clause: the result is well-defined when the definition is monotone (and also local, in the case of sheaf toposes).
} 
By a similar definition, it is possible to define an analogous operator in the internal type theory of $\mathcal{S}_{\odot}$, i.e. a fibered endofunctor - : $\mathcal{S}_{\odot} / X \times \mathbb{K} \rightarrow \mathcal{S}_{\odot} / X \times \mathbb{K}$; however, we have only needed the logical modality in our construction.

All the other forcing clauses are completely standard; for a reference on Kripke-Joyal forcing, see Mac Lane and Moerdijk [25].

\subsection{Programming language and operational semantics}

In Section 2 (Figure 2) we gave a grammar for the formal terms of CTT $_{\odot}$; however, in our semantics, we employ a second notion of syntax which is constructed as an inductive definition internal to $\mathcal{S}_{\odot}$; this is the language of programs, and differs from the syntax of formal terms in two respects:

1. Clocks in programs are imported directly from the metatheoretic object of clocks $\mathbb{K}: \mathcal{S}_{\odot}$; so the family of operators $\nabla_{\kappa}-$ is indexed in $\kappa: \mathbb{K}$ in exactly the same way that $U_{i}$ is indexed in $i: \mathbb{N}$.

2. The binding of clocks (such as in the clock intersection operator) is represented using the exponential $-{ }^{\mathbb{K}}: \mathcal{S}_{\odot} \rightarrow \mathcal{S}_{\odot}{ }^{6}$

Remark 1 (Generalized Syntax). The idea of using the exponential of the metalanguage in the syntax of a programming language is not new. Infinitary notions of program syntax can be traced back as far as Brouwer's $F$-inference in the justification of the Bar Principle [16], and have more recently been developed in Nuprl semantics [31], as well as in the context of higher-order focusing [37].

We will define the inductive family $\mathcal{P r o g}_{n}$ of programs with $n$ free variables in $\mathcal{S}_{\odot}$ using an internal inductive definition, summarized in Figure 3.

Our syntax forms a substitution algebra, and we write $\mathrm{p}_{(-)}$: $\mathcal{V a r}_{n} \rightarrow \mathcal{P r o g}_{n}$ for the injection of variables into terms; we write $M \cdot \gamma: \operatorname{Prog}_{n}$ for the action of the substitution $\gamma: \mathcal{P r o g}_{n} \operatorname{Var}_{m}$ in $M: \operatorname{Prog}_{m}$

Internal operational semantics Programs are endowed with operational meaning through the definition of a transition system, an illustrative fragment of which we present in Figure 3. This defines predicates - val $: \mathcal{P}\left(\mathcal{P r o g}_{0}\right)$ and $-\mapsto-: \mathcal{P}\left(\mathcal{P r o g}_{0} \times \mathcal{P r o g}_{0}\right)$ in $\mathcal{S}_{\odot}$. Write $\mathcal{V a l}: \mathcal{S}_{\odot}$ for the subobject $\left\{M: \mathcal{P r o g}_{0} \mid M\right.$ val $\}$.

Write $-\mapsto^{\star}-$ for the reflexive-transitive closure of $-\mapsto-$. We now define approximation and computational equivalence judgments $-\preccurlyeq-,-\approx-: \mathcal{P}\left(\mathcal{P r o g}_{0} \times \mathcal{P r o g}_{0}\right)$ respectively for closed programs as follows:

$$
\begin{aligned}
& M_{0} \preccurlyeq M_{1} \triangleq \forall M_{v}: \text { Val. } M_{0} \mapsto^{\star} M_{v} \Rightarrow M_{1} \mapsto^{\star} M_{v} \\
& M_{0} \approx M_{1} \triangleq M_{0} \preccurlyeq M_{1} \wedge M_{1} \preccurlyeq M_{0}
\end{aligned}
$$

The latter is extended to a computational equivalence judgment for open programs $-\approx_{n}-: \mathcal{P}\left(\mathcal{P r o g}_{n} \times \mathcal{P r o g}_{n}\right)$ by quantifying over total substitutions.

$$
M_{0} \approx_{n} M_{1} \triangleq \forall \gamma: \operatorname{Prog}_{0}^{n} \cdot M_{0} \cdot \gamma \approx M_{1} \cdot \gamma
$$

It would be desirable to extend this relation to a theory of computational congruence, as pioneered by Howe [23]; however, for our immediate purposes it has sufficed to require types only to respect the approximation relation defined above.

\footnotetext{
${ }^{6}$ While this construction cannot be called "ordinary syntax", it is an inductive definition that can be built up explicitly using the fact that $\mathcal{S}_{\odot}$ models indexed W-types [27].
}

Definition 2 (Computational PERs). A partial equivalence relation is a binary relation which is both symmetric and transitive. Such a relation $\mathcal{R}$ on $\operatorname{Prog}_{0}$ is called computational when it respects approximation in the following sense: if $\left(M_{0}, M_{1}\right) \in \mathcal{R}$ and $M_{0} \preccurlyeq$ $M_{0}^{\prime}$, then $\left(M_{0}^{\prime}, M_{1}\right) \in \mathcal{R}$.

Telescopes To capture the syntax of contexts and we define the inductive family $\mathcal{T}_{n}$ of telescopes of length $n$ as follows:

$$
\overline{\cdot: \mathcal{T}_{0}} \quad \frac{\Gamma: \mathcal{T}_{n} \quad A: \mathcal{P r o g}_{n}}{\Gamma . A: \mathcal{T}_{n+1}}
$$

Elaborating terms We now sketch the elaboration of the program terms of Section 2 into programs; approximately, a term $M$ with free formal clock variables $\Delta$ and free term variables $\Psi$ will be elaborated to a morphism $\|\Delta \mid \Psi \vdash M\|: \mathbb{K}^{|\Delta|} \rightarrow \operatorname{Prog}_{|\Psi|}$.

Notation 3. When $\Psi$ is a list, we write $|\Psi|$ for its length, and we write $\Psi[x]$ for the index $i<|\Psi|$ of the element $x$ in $\Psi$, presupposing $\Psi \ni x$.

We present here only a few of the most illustrative cases; the remainder of the elaboration can be found in technical report, and in our Coq formalization [34].

$$
\begin{aligned}
\|\Delta \mid \Psi \vdash x\| \varrho & =\mathrm{p}_{\Psi[x]} \\
\|\Delta \mid \Psi \vdash \lambda x . M\| \varrho & =\lambda(\|\varrho \mid \Psi, x \vdash M\| \varrho) \\
\|\Delta \mid \Psi \vdash \lambda k . M\| \varrho & =\lambda_{\odot}(\kappa \mapsto\|\Delta, k \mid \Psi \vdash M\|(\varrho, \kappa)) \\
\|\Delta \mid \Psi \vdash M k\| \varrho & =(\|\Delta \mid \Psi \vdash M\| \varrho)\left(\rho_{\Delta[k]}\right) \\
\left\|\Delta \mid \Psi \vdash \vdash_{k} A\right\| \varrho & =\bigodot_{\varrho_{\Delta}[k]}\|\Delta \mid \Psi \vdash A\| \varrho \\
\|\Delta \mid \Psi \vdash(k: \mathrm{clk}) \rightarrow A\| \varrho & =\Pi_{\odot}(\kappa \mapsto\|\Delta, k \mid \Psi \vdash A\|(\varrho, \kappa)) \\
\|\Delta \mid \Psi \vdash\{k \div \mathrm{clk}\} \rightarrow A\| \varrho & =\curvearrowleft(\kappa \mapsto\|\Delta, k \mid \Psi \vdash A\|(\varrho, \kappa))
\end{aligned}
$$

Elaborating contexts Next, we elaborate contexts $\Gamma$ with free formal clock variables $\Delta$ as morphisms $\|\Delta \mid \Gamma\|: \mathbb{K}^{|\Delta|} \rightarrow \mathcal{T}_{|\Gamma|}$, writing $\pi(\Gamma)$ for the sequence $\vec{x}_{i}$ when $\Gamma \equiv \overrightarrow{x_{i}: A_{i}}$.

$$
\begin{aligned}
\|\Delta \mid \cdot\| \varrho & =\cdot \\
\|\Delta \mid \Gamma, x: A\| \varrho & =(\|\Delta \mid \Gamma\| \varrho) .(\|\Delta \mid \pi(\Gamma) \vdash A\| \varrho)
\end{aligned}
$$

To save space, we may write $\|M\|$ or $\|\Gamma\|$ for the elaboration of a term or a context respectively, when the parameters are obvious.

\subsection{Full type system hierarchy}

At a high level, a type system in the sense of Allen [3] is an object which distinguishes some programs as types, and specifies what programs will be the elements of those types, and when they will be considered equal. Writing $\operatorname{rel}(X)$ for $\mathcal{P}(X \times X)$, we define a candidate type system to be a relation $\tau: \mathcal{P}\left(\mathcal{P r o g}_{0} \times \operatorname{rel}\left(\mathcal{P r o g}_{0}\right)\right)$ in $\mathcal{S}_{\odot}$. We will write $\mathrm{TS}_{\text {cand }}$ for the collection of such candidate type systems, i.e. $\operatorname{TS}_{\text {cand }}: \mathcal{S}_{\odot} \triangleq \mathcal{P}\left(\operatorname{Prog}_{0} \times \operatorname{rel}\left(\operatorname{Prog}_{0}\right)\right)$.

Let us now define notation for some assertions about candidate type systems $\tau: \mathrm{TS}_{\text {cand }}$ :

$$
\begin{gathered}
\tau \mid=A \doteq B \triangleq \exists \mathcal{A}: \operatorname{rel}\left(\operatorname{Prog}_{0}\right) .(A, \mathcal{A}) \in \tau \wedge(B, \mathcal{A}) \in \tau \\
\tau \mid=M_{0} \doteq M_{1} \in A \triangleq \exists \mathcal{A}: \operatorname{rel}\left(\mathcal{P r o g}_{0}\right) .(A, \mathcal{A}) \in \tau \wedge\left(M_{1}, M_{2}\right) \in \mathcal{A}
\end{gathered}
$$

A candidate type system $\tau$ : $\mathrm{TS}_{\text {cand }}$ can have the following characteristics:

1. It is called extensional if it is the graph of a partial function $\operatorname{Prog}_{0} \rightarrow \operatorname{rel}\left(\operatorname{Prog}_{0}\right)$. 


$$
\begin{aligned}
& \mathcal{V a r}_{n} \triangleq\{i \mid i<n\} \\
& \frac{i: \operatorname{Var}_{n}}{\mathrm{p}_{i}: \operatorname{Prog}_{n}} \quad \frac{M: \operatorname{Prog}_{n+1}}{\lambda(M): \operatorname{Prog}_{n}} \quad \frac{M: \operatorname{Prog}_{n}}{\lambda_{\odot}(M): \operatorname{Prog}_{n}} \quad \frac{M_{0}: \mathcal{P r o g}_{n} M_{1}: \mathcal{P r o g}_{n}}{M_{0}\left(M_{1}\right): \mathcal{P r o g}_{n}} \quad \frac{M: \mathcal{P r o g}_{n}}{M(\kappa): \mathcal{P r o g}_{n}} \quad \frac{\kappa: \mathbb{K}}{\operatorname{fix}_{(}(M): \mathcal{P r o g}_{n}}
\end{aligned}
$$

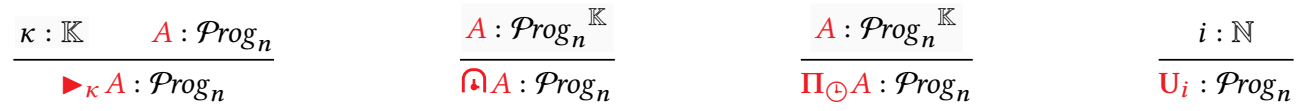

$$
\begin{aligned}
& \overline{\lambda(M) \text { val }} \quad \overline{{ }_{\kappa} A \text { val }} \quad \overline{\text { A } A \text { val }} \quad \overline{\mathrm{U}_{i} \text { val }} \quad \frac{M_{0} \mapsto M_{0}^{\prime}}{M_{0}\left(M_{1}\right) \mapsto M_{0}^{\prime}\left(M_{1}\right)} \quad \overline{\left(\lambda\left(M_{f}\right)\right)(M) \mapsto M_{f} \cdot M} \quad \overline{\operatorname{fix}(M) \mapsto M \cdot \operatorname{fix}(M)}
\end{aligned}
$$

Figure 3. An illustrative fragment of the inductive definition of the programs with $n$ free variables $\mathcal{P r o g}_{n}: \mathcal{S}_{\odot}$, and their operational semantics. For the full definition, see either our report [33] or our Coq formalization [34].

2. It is called computational PER-valued if whenever $(A, \mathcal{A}) \in \tau$, the relation $\mathcal{A}$ is a computational PER (see Definition 2).

3. It is called type-computational when, if $(A, \mathcal{A}) \in \tau$ and $A \preccurlyeq$ $A^{\prime}$, then also $\left(A^{\prime}, \mathcal{A}\right) \in \tau$.

Finally a candidate type system is called a type system if it is extensional, computational PER-valued, and type-computational. We write TS : $\mathcal{S}_{\odot}$ for the collection of such type systems.

Sequents and functionality Next, we briefly sketch the meaning of type functionality sequents $\Gamma \gg A_{0} \doteq A_{1}$ and functionality sequents $\Gamma \gg M_{0} \doteq M_{1} \in A$ using a simple notion of functionality derived from Martin-Löf [26], with respect to any candidate type system $\tau: \mathrm{TS}_{\text {cand }}$.

When $\Gamma: \mathcal{T}_{n}$ is a telescope and $\gamma_{0}, \gamma_{1}: \mathcal{P r o g}_{0}^{n}$ are sequences of programs, we define similarity of instantiations $\gamma_{0} \doteq \gamma_{1} \in^{\star} \Gamma$ by recursion on $\Gamma . \cdot \doteq \cdot \epsilon^{\star}$. is true, and $\gamma_{0} \cdot M_{0} \doteq \gamma_{1} \cdot M_{1} \in^{\star} \Gamma \cdot A$ is true when both $\gamma_{0} \doteq \gamma_{1} \in^{\star} \Gamma$ and $M_{0} \cdot \gamma_{0} \doteq M_{1} \cdot \gamma_{1} \in A \cdot \gamma_{0}$ are true.

Open type similarity $\Gamma \gg A_{0} \doteq A_{1}$ is true when for all instantiations $\gamma_{0} \doteq \gamma_{1} \in^{\star} \Gamma$, we have $A_{0} \cdot \gamma_{0} \doteq A_{1} \cdot \gamma_{1}$. Likewise, open member smilarity $\Gamma \gg M_{0} \doteq M_{1} \in A$ is true when for all such instantiations, we have $M_{0} \cdot \gamma_{0} \doteq M_{1} \cdot \gamma_{1} \in A \cdot \gamma_{0}$.

Finally, context validity $\Gamma$ ctx is given by recursion on $\Gamma$ using open type similarity in the inductive case.

\subsection{Closure under type formers other than universes}

Next, we will show how to close a candidate type system under the type formers of $\mathbf{C T T}_{\odot}$, namely booleans, natural numbers, dependent functions types, dependent pair types, equality types, later modalities, clock intersection types and universes.

The simplest way to carry out this construction, as pioneered by Crary [19] and formalized by Anand and Rahli [4], is to use an inductive definition of a closure operator $\mathrm{c}[-]: \mathrm{TS}_{\text {cand }} \rightarrow \mathrm{TS}_{\text {cand }}$ on candidate type systems. However, this method does not immediately extend to the type systems that we consider in this paper, because it is not clear how to fit the clause for the later modality into the usual schemata for inductive definitions based on strictly positive signatures.

Therefore, as advocated by Allen [3], we will build up our closure operator manually by taking the least fixed point of a monotone operator on candidate type systems; this construction can be carried out in any topos, because the Knaster-Tarski theorem guarantees a least fixed point for any monotone operator on a complete lattice [21].

First, we define some notation for closing relations and type systems under evaluation to canonical form:

$$
\begin{aligned}
& -\Downarrow: \operatorname{rel}\left(\mathcal{P r o g}_{0}\right) \rightarrow \operatorname{rel}\left(\mathcal{P r o g}_{0}\right) \\
& \mathcal{A}^{\Downarrow} \triangleq\left\{\left(M_{0}, M_{1}\right) \mid \exists M_{0}^{v}, M_{1}^{v}: \text { Val. } M_{i} \mapsto^{\star} M_{i}^{v} \wedge\left(M_{0}^{v}, M_{1}^{v}\right) \in \mathcal{A}\right\} \\
& -\Downarrow: \operatorname{TS}_{\text {cand }} \rightarrow \operatorname{TS}_{\text {cand }} \\
& \tau^{\Downarrow} \triangleq\left\{(A, \mathcal{A}) \mid \exists A_{v}: \text { Val. } A \mapsto^{\star} A_{v} \wedge\left(A_{v}, \mathcal{A}\right) \in \tau\right\}
\end{aligned}
$$

In Figure 4, for an initial candidate type system $\sigma: \mathrm{TS}_{\text {cand }}$, we define an endomorphism on candidate type systems $\mathfrak{\Im}_{\sigma}: \mathrm{TS}_{\text {cand }} \rightarrow \mathrm{TS}_{\text {cand }}$ which extends a type system with all the non-universe connectives of $\mathbf{C T T}_{\odot}$.

Theorem 4 (Closure. Clo. monotonicity). For any candidate type system $\sigma: \mathrm{TS}_{\text {cand }}$, the function $\tilde{F}_{\sigma}: \mathrm{TS}_{\text {cand }} \rightarrow \mathrm{TS}_{\text {cand }}$ is monotone.

Proof. By case on the type closure clauses above, which are themselves each monotone.

Corollary 5 (Closure. Clo. t, Closure. Clo. roll). By the KnasterTarski theorem, the function $\mathfrak{\mho}_{\sigma}$ has a least fixed point $\mu\left(\mathfrak{\mho}_{\sigma}\right)$.

We will write $\mathbf{c}[-]: \mathrm{TS}_{\text {cand }} \rightarrow \mathrm{TS}_{\text {cand }}$ for the operator that takes $\sigma: \mathrm{TS}_{\text {cand }}$ to the fixed point $\mu\left(\mathfrak{F}_{\sigma}\right)$.

\subsection{The full universe hierarchy}

The next step in the construction is to build up the universe hierarchy. Following Allen [3], we define the "spine" of the universe hierarchy as a sequence of type systems $v: \mathrm{TS}_{\text {cand }}^{\mathbb{N}}$ that contains at each level only types which evaluate to universes:

$$
\begin{aligned}
v_{0} & =\perp \\
v_{n+1} & =\left\{\left(\mathrm{U}_{i}, \mathcal{U}\right) \mid i \leq n \wedge \mathcal{U} \equiv\left\{\left(A_{0}, A_{1}\right)\left|\mathbf{c}\left[v_{i}\right]\right|=A_{0} \doteq A_{1}\right\}\right\}^{\Downarrow}
\end{aligned}
$$

The sequence above is well-defined by complete induction on the index. We are now equipped to define a new sequence of type systems which is at each level closed under all the ordinary type formers as well as smaller universes:

$$
\tau_{n} \triangleq \mathbf{c}\left[v_{n}\right]
$$




$$
\begin{aligned}
& \mathfrak{\mho}_{\sigma}: \mathrm{TS}_{\text {cand }} \rightarrow \mathrm{TS}_{\text {cand }} \\
& \mathfrak{F}_{\sigma}(\tau) \triangleq \sigma \cup(\operatorname{VoId}(\tau) \cup \operatorname{UNIT}(\tau) \cup \operatorname{BoOL}(\tau) \cup \operatorname{Nat}(\tau) \cup \operatorname{Prod}(\tau) \cup \operatorname{Fun}(\tau) \cup \operatorname{EQ}(\tau) \cup \operatorname{Ltr}(\tau) \cup \operatorname{Isect}(\tau) \cup \operatorname{KFUN}(\tau) \cup \operatorname{TrEE}(\tau))^{\Downarrow} \\
& \operatorname{LTR}(\tau) \triangleq\left\{\left(\triangleright_{\kappa} A, \mathcal{X}\right) \mid \exists \mathcal{A}: \operatorname{rel}\left(\operatorname{Prog}_{0}\right) \cdot \triangleright_{\kappa}((A, \mathcal{A}) \in \tau) \wedge \mathcal{X} \equiv\left\{\left(M_{0}, M_{1}\right) \mid \triangleright_{\kappa}\left(\left(M_{0}, M_{1}\right) \in \mathcal{A}\right)\right\}\right\} \\
& \left.\operatorname{Isect}(\tau) \triangleq\left\{(\curvearrowright A, \mathcal{X}) \mid \exists \mathcal{A}: \operatorname{rel}\left(\operatorname{Prog}_{0}\right)\right)^{\mathbb{K}} .(\forall \kappa: \mathbb{K} .(A(\kappa), \mathcal{A}(\kappa)) \in \tau) \wedge \mathcal{X} \equiv\left\{\left(M_{0}, M_{1}\right) \mid \forall \kappa: \mathbb{K} .\left(M_{0}, M_{1}\right) \in \mathcal{A}(\kappa)\right\}\right\} \\
& \operatorname{KFuN}(\tau) \triangleq\left\{\left(\Pi_{\odot} A, \mathcal{X}\right) \mid \exists \mathcal{A}: \operatorname{rel}\left(\mathcal{P r o g}_{0}\right)^{\mathbb{K}} .(\forall \kappa: \mathbb{K} .(A(\kappa), \mathcal{A}(\kappa)) \in \tau) \wedge \mathcal{X} \equiv\left\{\left(M_{0}, M_{1}\right) \mid \forall \kappa: \mathbb{K} .\left(M_{0}(\kappa), M_{1}(\kappa)\right) \in \mathcal{A}(\kappa)\right\}\right\}
\end{aligned}
$$

Figure 4. A monotone operator on candidate type systems; for the sake of space, we elide the interpretations of the standard connectives. For the remainder, please see our report [33].

Finally, we can capture the entire countable hierarchy in a single type system $\tau_{\omega}$, which is the join of the entire sequence:

$$
\tau_{\omega} \triangleq \bigvee_{i: \mathbb{N}} \tau_{i}
$$

When we explain the meaning of judgments, it will always be done with respect to this maximal type system.

Theorem 6 ( $\tau_{\omega}$ type system). The ultimate candidate type system $\tau_{\omega}$ is in fact a type system.

\subsection{Meaning explanation}

In this section, we give a mathematical meaning explanation to the formal judgments of $\mathbf{C T T}_{\odot}$ :

1. Functional equality of elements $\Delta \mid \Gamma \gg M_{0} \doteq M_{1} \in A$ means that in clock context $\Delta$ and variable context $\Gamma, M_{0}$ and $M_{1}$ are equal elements of type $A$. This form of judgment requires that $\Gamma, M_{0}, M_{1}, A$ mention only clocks from $\triangle$, and that $M_{0}, M_{1}, A$ mention only variables from $\Gamma$.

2. Untyped open conversion $\Delta \mid \Psi \vdash M_{0} \leftrightarrow M_{1}$ means that $M_{0}$ and $M_{1}$ are Kleene equivalent in all their instantiations. This form of judgment requires that $M_{0}, M_{1}$ mention only clocks from $\Delta$ and variables from $\Psi$.

The meaning of judgments We interpret each formal judgment $\mathcal{J}$ as a proposition $\llbracket \mathcal{J} \rrbracket: \Omega$ in $\mathcal{S}_{\odot}$.

$$
\begin{aligned}
& \llbracket \Delta \mid \Gamma \gg M_{0} \doteq M_{1} \in A \rrbracket \triangleq \\
& \forall \varrho: \mathbb{K}^{|\Delta|} . \\
& \tau_{\omega} \mid=\|\Gamma\| \varrho \operatorname{ctx} \\
& \Rightarrow \tau_{\omega} \mid=\|\Gamma\| \varrho \gg\left\|A_{0}\right\| \varrho \doteq\left\|A_{1}\right\| \varrho \\
& \Rightarrow \tau_{\omega} \mid=\|\Gamma\| \varrho \gg\left\|M_{0}\right\| \varrho \doteq\left\|M_{1}\right\| \varrho \in\|A\| \varrho \\
& \llbracket \Delta\left|\Psi \vdash M_{0} \leftrightarrow M_{1} \rrbracket \triangleq \forall \varrho: \mathbb{K}^{|\Delta|} .\left\|M_{0}\right\| \varrho \approx\right| \Psi \mid\left\|M_{1}\right\| \varrho
\end{aligned}
$$

Observe that the usual presuppositions of the equality judgment (context validity and type functionality) are taken as assumptions: the principle can be summarized as "garbage in, garbage out". Dually, we could have chosen to regard them as consequences, which would lead to a slightly different collection of validated rules.

Canonicity at base type Write $2: \mathcal{S}_{\odot}$ for the boolean object in our semantic framework which has two global elements $2_{0}, 2_{1}: 2$. Define an embedding $L-\rfloor_{2}$ from this object into our formal term language as follows:

$$
\begin{aligned}
& \left\lfloor 2_{0}\right\rfloor_{2}=t t \\
& \left\lfloor 2_{1}\right\rfloor_{2}=f f
\end{aligned}
$$

Now we can state the canonicity theorem for $\mathbf{C T T}_{\odot}$.

Theorem 7 (Canonicity. canonicity). For any closed expression $M$ such that $\llbracket \cdot \mid \cdot \gg M \doteq M \in$ bool $\rrbracket$, there exists some $b \in 2$ such that $\llbracket \cdot \mid \cdot \vdash M \leftrightarrow\lfloor b\rfloor_{2} \rrbracket$.

Corollary 8. The type theory $\mathbf{C T T}_{\odot}$ is consistent in the sense that there is no inhabitant of void.

\subsection{Validated rules}

In Figure 5, we present a small selection of the rules which we have validated in our Coq formalization of $\mathbf{C T T}_{\odot}$; we omit most of the standard rules of ordinary extensional type theory, which are also valid in our semantics.

\subsection{Examples: revisiting streams}

Using these rules, we can derive some typing lemmas for guarded streams and coinductive sequences of bits.

$$
\begin{aligned}
\text { stream } & \triangleq \lambda k \text {. fix } A \text { in bool } \times{ }_{k} A \\
\text { sequence } & \triangleq\{k \div \mathrm{clk}\} \rightarrow \text { stream } k \\
\text { ones } & \triangleq \mathrm{fix} x \text { in }\langle\mathrm{tt}, x\rangle
\end{aligned}
$$

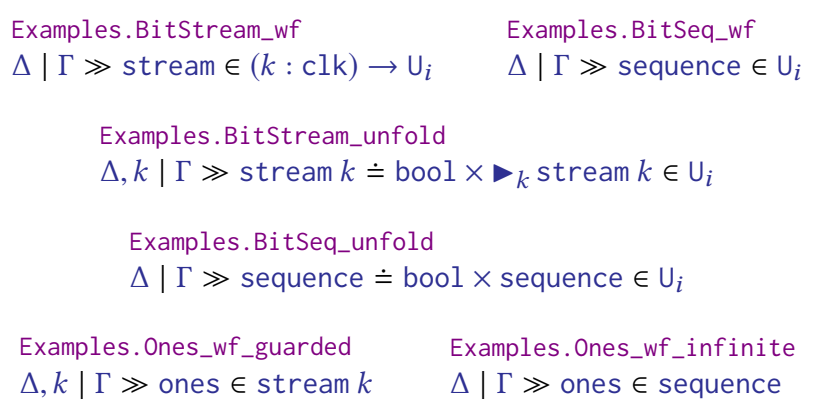

\section{Survey of Related Work}

\subsection{Guarded Dependent Type Theory}

The standard model of guarded recursion without clocks is the topos of trees $\widehat{\omega}$, the presheaves on the poset of natural numbers regarded as a category [9]. This topos can be regarded as a denotational model for a variant of Martin-Löf's extensional type theory equipped with the modality. By indexing this topos over a category of clock contexts $\Delta$, it is possible to develop a model of extensional type 


$$
\begin{aligned}
& \text { Isect. univ_eq } \\
& \Delta, k \mid \Gamma \gg A_{0} \doteq A_{1} \in U_{i} \\
& \Delta \mid \Gamma \gg\{k \div \mathrm{clk}\} \rightarrow A_{0} \doteq\{k \div \mathrm{clk}\} \rightarrow A_{1} \in \mathrm{U}_{i} \\
& \text { Isect.intro } \\
& \frac{\Delta, k\left|\Gamma \gg M_{0} \doteq M_{1} \in A \quad \Delta, k\right| \Gamma \gg A \in \mathrm{U}_{i}}{\Delta \mid \Gamma \gg M_{0} \doteq M_{1} \in\{k \div \mathrm{clk}\} \rightarrow A}
\end{aligned}
$$

Figure 5. A selection of the rules which we have proved correct for our type theory.

theory with clock quantification called GDTT $[12,13]$. In order to justify a crucial clock irrelevance principle, it is necessary to index universes in clock contexts, i.e. $\mathcal{U}_{\Delta}$.

In the dependent setting, some difficulties arise when devising a syntax for the semantic type theory of this indexed category. In order to make sense of the "delayed application" operator $\circledast$ in the context of dependent function types, it was necessary to introduce a notion of delayed substitution $\xi \equiv[\overrightarrow{x \leftarrow e}]$ which pervades the term language, introducing term formers like $\triangleright^{k} \xi . A$ and next ${ }^{k} \xi$.e. On the bright side, delayed application can be defined in terms of delayed substitution.

However, the equational theory for delayed substitutions is fairly sophisticated, and an operational (computational) interpretation of GDTT has not yet been proposed at the time this article was written; as such, a canonicity theorem for this system is still forthcoming.

\subsection{Orthogonality and clock irrelevance}

In a more recent development [14], a denotational model of GDTT has been developed that differs from that of Bizjak and Møgelberg [13] in a few crucial ways.

Unified base category The fibered topos presentation of the Bizjak and Møgelberg [13] work has been replaced with a presheaf topos over a single unified base category, discovered independently from the unified base category which we introduce in Section 3.1. Taking presheaves over this unified base category simplifies the model significantly, and also makes available the standard solution to the substitution coherence problem for (denotational) presheaf models of dependent type theory. ${ }^{7}$

The proposed base category of Bizjak and Møgelberg [14] differs from ours mainly in that they allow empty worlds, whereas we restrict our base category to those worlds which contain at least a single clock.

Orthogonality Bizjak and Møgelberg define a presheaf of clocks $C$ which is the same as our object of clocks $\mathbb{K}$ which we introduce in Section 3.1; then, the clock quantifier is represented in the internal language of their presheaf topos as a dependent product over $C$, i.e. $\prod_{x: C} A(x)$.

Defined in this way, the clock quantifier cannot be a priori parametric with respect to clocks / time objects; therefore, in order to validate the clock irrelevance axiom, the authors have identified an orthogonality condition on objects, which in essence closes the internal language of the presheaf topos under just those types

\footnotetext{
${ }^{7}$ This is to use an alternative construction of the slice categories $\widehat{\mathbb{C}} / X$, as the presheaves on the total category of $X$.
} 
which are compatible with the irrelevance principle for the clock quantifier.

Unfortunately, the subtopos of time-orthogonal objects does not contain the standard Hofmann-Streicher universes, because universes necessarily classify types that depend on clocks in an essential way. In order to resolve this problem, the standard presheaftheoretic universe $\mathcal{U}$ is replaced with a family of universes $\mathcal{U}_{\Delta}$ for each clock context $\Delta$; each universe $\mathcal{U}_{\Delta}$ classifies the types which may depend only on the clocks in $\Delta$.

Discussion Temporarily abstracting away from the differences between a denotational account of GDTT and our operational account of type theory, we can briefly summarize the difference between our approaches to clock quantification and irrelevance.

The approach of Bizjak and Møgelberg [14] is in essence to define clock quantification as a dependent (cartesian) product, and then restrict the available semantic constructions to precisely those which treat clocks parametrically; then, within this subcategory, the clock quantifier can itself be regarded as a parametric quantifier (because all counterexamples have been muted).

Our approach is instead to define clock quantifiers which intrinsically behave in the desired way, rather than starting with only a proof-relevant quantifier and ruling out observations of its non-parametric character using a global orthogonality condition. To that end, we have defined two separate clock quantifiers which decompose the two disjoint uses of $\forall \kappa$ from GDTT:

1. A parametric quantifier $\{k \div \mathrm{clk}\} \rightarrow A$ for expressing that a program exhibits a behavior relative to all clocks simultaneously. Semantically, this is an intersection, though we expect that a more refined perspective will arise as we explore other kinds of model where the intersection may not be available.

2. A non-parametric quantifier $(k: \mathrm{clk}) \rightarrow A$ for internalizing a family of objects which varies in a clock; semantically this is the cartesian product of a clock-indexed family of types (i.e. the right adjoint to weakening). A priori there is no need for this quantifier to behave parametrically, as this is neither demanded nor desired when forming families of objects.

In this way, we have managed to avoid imposing any global orthogonality condition on the objects of our semantic model, leading to a smoother treatment of universes that avoids indexing in clock contexts.

\subsection{Guarded Cubical Type Theory}

One way to achieve a decidable typing judgment for GDTT is to adopt an intensional equality, and replace various judgmental principles with propositional axioms (such as the unfolding rule for fix, as well as several other principles having to do with identity types which are validated in extensional GDTT). However, such axioms are disruptive to the computational character of type theory.

A more refined and well-behaved version of this idea can be found in Guarded Cubical Type Theory (GCTT) by Birkedal et al. [8], where fix is actually exhibited as a higher-dimensional term, a line or path between a formal fixed point and its one-step unfolding.

GCTT currently supports only a single clock, but it is plausible that it could be extended in the same way as GDTT extends the internal type theory of the topos of trees. Although GCTT does not at the time of writing have a decidable typing result, nor a strong normalization theorem, we are confident that these can be achieved in the future in light of the intensional judgmental equality and the restricted unfoldings of fixed points.

\subsection{Clocked Type Theory}

Recently, an alternative to GDTT called Clocked Type Theory (CloTT) has been proposed, which enjoys a computational interpretation with a canonicity result [7]; it is plausible that Clocked Type Theory shall have a decidable typing relation. Notably, Clocked Type Theory does not validate any clock irrelevance rule; the authors propose to address this in a cubical version of CloTT by adding a special path axiom which realizes this principle, by analogy with the technique used in GCTT to account for restricted unfoldings of fixed points. In the presence of this axiom, canonicity for CloTT can still be made to hold in the context which contains only a single clock.

Discussion Clocked Type Theory looks like a promising path toward a well-behaved intrinsic account of guarded recursion with clocks. In the present paper, our efforts have been focused exclusively on developing the behavioral account of guarded type theory in the style of Martin-Löf's meaning explanation, in which programs can be regarded as existing separately from their types; here, general recursive programs can be written and shown to be (causal, productive, total) in a semantic sense, using the type theory as a program logic.

We perceive, however, that virtue lies in pursuing the intrinsic path, especially as far as implementability are concerned. The calculus developed in Bahr et al. [7] (and more recently, the ideas contained in Clouston et al. [17]) are likely to provide the basis for a syntactic account of guarded recursion which is sound for our model, but closer to implementation.

\subsection{Sized Types and size quantifiers}

Our decomposition of the quantifier $\forall \kappa$ from GDTT into a parametric part $\{k \div \mathrm{clk}\} \rightarrow A$ and a non-parametric part $(k: \mathrm{clk}) \rightarrow A$ mirrors the state of affairs in the literature on sized types, which is another account of type-based guarded recursion [1].

\section{Perspective and Future Work}

We have developed and formalized a computational account of guarded dependent type theory with clocks, enjoying several desirable characteristics not found together in other existing models: computational canonicity, clock irrelevance and ordinary universes. We have made the following contributions toward a simpler, more computational account of guarded dependent type theory:

Implementation, proof theory, and syntax We have not yet tackled the project of developing an ergonomic proof theory for $\mathbf{C T T}_{\odot}$ which can be used to interact with the semantics presented here. The natural deduction style rules which we have given here are, while convenient for paper presentations, not what one would use in a serious implementation. To build a proof theory for $\mathbf{C T T}_{\odot}$, we must negotiate new forms of judgment with decidable presupposition.

Therefore, while we have indeed developed a programming language for guarded type theory with clocks that omits explicit syntax for delayed substitutions, this should be understood in terms of the conceptual order of semantics and proof theory which is endemic in computational type theory. In particular, while our programming 
language and type theory has no need for such a construct, in a proof language for $\mathbf{C T T}_{\odot}$ it would be necessary to account for the syntactic structure of the later modality's elimination; we anticipate that ideas from Bahr et al. [7] and Clouston et al. [17] will be highly relevant.

Application to denotational semantics In the future, we are interested in extending our work to a denotational account of guarded dependent type theory with clocks which uses the ordinary nonindexed presheaf-topos-theoretic universe. While our results have been developed in the context of computational type theory and operational semantics, we believe that the insight which enabled us to combine clock irrelevance with ordinary universes is more broadly applicable.

\section{Acknowledgments}

We are thankful to Carlo Angiuli, Lars Birkedal, Aleš Bizjak, Jonas Frey, Daniel Gratzer, Adrien Guatto, Pieter Hofstra, Bas Spitters, Sam Staton, and Joseph Tassarotti for helpful discussions on the semantics of guarded recursion, clock names and universe hierarchies. Thanks to David Christiansen for his comments on a draft of this paper.

The authors gratefully acknowledge the support of the Air Force Office of Scientific Research through MURI grant FA9550-15-10053. Any opinions, findings and conclusions or recommendations expressed in this material are those of the authors and do not necessarily reflect the views of the AFOSR.

\section{References}

[1] Andreas Abel, Andrea Vezzosi, and Theo Winterhalter. 2017. Normalization by Evaluation for Sized Dependent Types. Proc. ACM Program. Lang. 1, ICFP, Article 33 (Aug. 2017), 30 pages.

[2] S.F. Allen, M. Bickford, R.L. Constable, R. Eaton, C. Kreitz, L. Lorigo, and E. Moran 2006. Innovations in computational type theory using Nuprl. Fournal of Applied Logic 4, 4 (2006), 428 - 469. Towards Computer Aided Mathematics.

[3] Stuart Frazier Allen. 1987. A non-type-theoretic semantics for type-theoretic language. Ph.D. Dissertation. Cornell University, Ithaca, NY, USA.

[4] Abhishek Anand and Vincent Rahli. 2014. Towards a Formally Verified Proof Assistant. In Interactive Theorem Proving: 5th International Conference, ITP 2014, Held as Part of the Vienna Summer of Logic, VSL 2014, Vienna, Austria, Fuly 14-17, 2014. Proceedings, Gerwin Klein and Ruben Gamboa (Eds.). Springer International Publishing, Cham, 27-44.

[5] Andrew W. Appel, Paul-André Melliès, Christopher D. Richards, and Jérôme Vouillon. 2007. A Very Modal Model of a Modern, Major, General Type System. In Proceedings of the 34th Annual ACM SIGPLAN-SIGACT Symposium on Principles of Programming Languages (POPL '07). ACM, New York, NY, USA, 109-122.

[6] Robert Atkey and Conor McBride. 2013. Productive Coprogramming with Guarded Recursion. In Proceedings of the 18th ACM SIGPLAN International Conference on Functional Programming (ICFP '13). ACM, New York, NY, USA, 197-208.

[7] P. Bahr, H. B. Grathwohl, and R. E. Møgelberg. 2017. The clocks are ticking: No more delays!. In 2017 32nd Annual ACM/IEEE Symposium on Logic in Computer Science (LICS). 1-12.

[8] Lars Birkedal, Aleš Bizjak, Ranald Clouston, Hans Bugge Grathwohl, Bas Spitters, and Andrea Vezzosi. 2016. Guarded Cubical Type Theory: Path Equality for Guarded Recursion. In 25th EACSL Annual Conference on Computer Science Logic (CSL 2016) (Leibniz International Proceedings in Informatics (LIPIcs)), Jean-Marc Talbot and Laurent Regnier (Eds.), Vol. 62. Schloss Dagstuhl-Leibniz-Zentrum fuer Informatik, Dagstuhl, Germany, 23:1-23:17.

[9] Lars Birkedal, Rasmus Ejlers Møgelberg, Jan Schwinghammer, and Kristian Stovring. 2011. First Steps in Synthetic Guarded Domain Theory: Step-Indexing in the Topos of Trees. In Proceedings of the 2011 IEEE 26th Annual Symposium on Logic in Computer Science (LICS '11). IEEE Computer Society, Washington, DC, USA, 55-64.

[10] Aleš Bizjak. 2016. On semantics and applications of guarded recursion. Ph.D. Dissertation. University of Aarhus.

[11] Aleš Bizjak, Lars Birkedal, and Marino Miculan. 2014. A Model of Countable Nondeterminism in Guarded Type Theory. In Rewriting and Typed Lambda Calculi, Gilles Dowek (Ed.). Springer International Publishing, Cham, 108-123.

[12] Aleš Bizjak, Hans Bugge Grathwohl, Ranald Clouston, Rasmus E. Møgelberg, and Lars Birkedal. 2016. Guarded Dependent Type Theory with Coinductive Types.
In Foundations of Software Science and Computation Structures: 19th International Conference, FOSSACS 2016, Held as Part of the European foint Conferences on Theory and Practice of Software, ETAPS 2016, Eindhoven, The Netherlands, April 2-8, 2016, Proceedings, Bart Jacobs and Christof Löding (Eds.). Springer Berlin Heidelberg, Berlin, Heidelberg, 20-35.

[13] Aleš Bizjak and Rasmus Ejlers Møgelberg. 2015. A Model of Guarded Recursion With Clock Synchronisation. Electron. Notes Theor. Comput. Sci. 319, C (Dec. 2015), 83-101.

[14] Aleš Bizjak and Rasmus Ejlers Møgelberg. 2017. Denotational semantics for guarded dependent type theory. (2017). Draft.

[15] Edwin Brady. 2013. Idris, a general-purpose dependently typed programming language: Design and implementation. Fournal of Functional Programming 23, 5 (Sep 2013), 552-593.

[16] L. E. J. Brouwer. 1981. Brouwer's Cambridge Lectures on Intuitionism. Cambridge University Press.

[17] Ranald Clouston, Bassel Mannaa, Rasmus Ejlers Møgelberg, Andrew M. Pitts, and Bas Spitters. 2018. Modal Dependent Type Theory and Dependent Right Adjoints. (2018). https://arxiv.org/abs/1804.05236.

[18] R. L. Constable, S. F. Allen, H. M. Bromley, W. R. Cleaveland, J. F. Cremer, R. W. Harper, D. J. Howe, T. B. Knoblock, N. P. Mendler, P. Panangaden, J. T. Sasaki, and S. F. Smith. 1986. Implementing Mathematics with the Nuprl Proof Development System. Prentice-Hall, Inc., Upper Saddle River, NJ, USA.

[19] Karl Crary. 1998. Type-Theoretic Methodology for Practical Programming Languages. Ph.D. Dissertation. Cornell University, Ithaca, NY.

[20] R.L. Crole. 1993. Categories for Types. Cambridge University Press, New York.

[21] Brian A. Davey and Hilary A. Priestley. 1990. Introduction to lattices and order. Cambridge University Press, Cambridge.

[22] Murdoch J. Gabbay and Martin Hofmann. 2008. Nominal Renaming Sets. In Proceedings of the 15th International Conference on Logic for Programming, Artificial Intelligence, and Reasoning (LPAR '08). Springer-Verlag, Berlin, Heidelberg, 158-173.

[23] Douglas J. Howe. 1989. Equality in Lazy Computation Systems. In Proceedings of Fourth IEEE Symposium on Logic in Computer Science. IEEE Computer Society, New York, 198-203.

[24] Ralf Jung, David Swasey, Filip Sieczkowski, Kasper Svendsen, Aaron Turon, Lars Birkedal, and Derek Dreyer. 2015. Iris: Monoids and Invariants As an Orthogonal Basis for Concurrent Reasoning. In Proceedings of the 42Nd Annual ACM SIGPLANSIGACT Symposium on Principles of Programming Languages (POPL '15). ACM, New York, NY, USA, 637-650.

[25] Saunders Mac Lane and Ieke Moerdijk. 1992. Sheaves in geometry and logic : a first introduction to topos theory. Springer, New York.

[26] Per Martin-Löf. 1979. Constructive Mathematics and Computer Programming. In 6th International Congress for Logic, Methodology and Philosophy of Science. Hanover, 153-175. Published by North Holland, Amsterdam. 1982.

[27] Ieke Moerdijk and Erik Palmgren. 2000. Wellfounded trees in categories. Annals of Pure and Applied Logic 104, 1 (2000), 189 - 218.

[28] H. Nakano. 2000. A modality for recursion. In Proceedings Fifteenth Annual IEEE Symposium on Logic in Computer Science (Cat. No.99CB36332). IEEE Computer Society, New York, 255-266.

[29] Ulf Norell. 2009. Dependently Typed Programming in Agda. In Proceedings of the 4th International Workshop on Types in Language Design and Implementation (TLDI '09). ACM, New York, NY, USA, 1-2.

[30] Marco Paviotti, Rasmus Ejlers MÃ̈̈gelberg, and Lars Birkedal. 2015. A Model of PCF in Guarded Type Theory. Electronic Notes in Theoretical Computer Science 319, Supplement C (2015), 333 - 349. The 31st Conference on the Mathematical Foundations of Programming Semantics (MFPS XXXI).

[31] Vincent Rahli, Mark Bickford, and Robert Constable. 2017. Bar induction: The good, the bad, and the ugly. In 2017 32nd Annual ACM/IEEE Symposium on Logic in Computer Science (LICS). 1-12.

[32] Sam Staton. 2007. Name-passing process calculi: operational models and structural operational semantics. Technical Report UCAM-CL-TR-688. University of Cambridge, Computer Laboratory.

[33] Jonathan Sterling and Robert Harper. 2018. Guarded Computational Type Theory (Technical Report). (2018). https://arxiv.org/abs/1804.09098

[34] Jonathan Sterling and Robert Harper. 2018. coq-guarded-type-theory. (2018). https://github.com/jonsterling/coq-guarded-type-theory

[35] The Coq Development Team. 2016. The Coq Proof Assistant Reference Manual. (2016).

[36] Andrea Vezzosi. 2015. Guarded Recursive Types in Type Theory. Institutionen för data- och informationsteknik, Datavetenskap (Chalmers), Chalmers tekniska högskola. 63.

[37] Noam Zeilberger. 2009. The logical basis of evaluation order and pattern-matching. Ph.D. Dissertation. Carnegie Mellon University. 